\title{
Factors associated with COVID-19 Vaccine Hesitancy in Thai Seniors
}

\author{
Saran Thanapluetiwong (D) \\ Sirintorn Chansirikarnjana (iD ${ }^{\prime}$ \\ Orapitchaya Sriwannopas' \\ Taweevat Assavapokee' \\ Pichai Ittasakul (D) ${ }^{2}$ \\ 'Division of Geriatric Medicine, \\ Department of Medicine, Faculty of \\ Medicine, Ramathibodi Hospital, Mahidol \\ University, Bangkok, Thailand; \\ ${ }^{2}$ Department of Psychiatry, Faculty of \\ Medicine, Ramathibodi Hospital, Mahidol \\ University, Bangkok, Thailand
}

Correspondence: Pichai Ittasakul Department of Psychiatry, Faculty of Medicine Ramathibodi Hospital, Mahidol University, 270 Rama VI Road, Ratchathewi, Bangkok, 10400, Thailand Tel +66 2-20l-1235

Fax +66 2-354-7299

Email pichail 18@gmail.com
Objective: Older people are the most vulnerable group for developing SARS-CoV-2 infection. Although vaccination against coronavirus disease 2019 (COVID-19) reduces infection, hospitalization, and mortality rates, some older people have refused to get vaccinated. Our study aimed to evaluate factors associated with COVID-19 vaccine hesitancy among Thai seniors.

Methods: We conducted a cross-sectional telephone survey on vaccine hesitancy in a geriatric clinic at Ramathibodi Hospital in Bangkok, Thailand. Patients aged $\geq 60$ years were contacted and interviewed by trained interviewers between June 20 and July 25, 2021. Results: In total, we interviewed 282 participants aged 60-93 years (mean age 73.0 \pm 7.5 years). We found that $44.3 \%$ of participants were hesitant to get a COVID-19 vaccination. Factors associated with high vaccine hesitancy were low education, lack of confidence in the healthcare system's ability to treat patients with COVID-19, vaccine manufacturers, being offered a vaccine from an unexpected manufacturer, and a low number of new COVID-19 cases per day.

Conclusion: The prevalence of COVID-19 vaccine hesitancy among Thai seniors is relatively high, and is associated with specific factors. These findings will help in promoting COVID-19 vaccination among Thailand's senior citizens.

Keywords: vaccine acceptance, vaccine refusal, elderly, older adult, SARS-CoV-2

\section{Introduction}

As of the end of June 2021, over 180 million people worldwide have suffered from severe acute respiratory syndrome coronavirus 2 (SARS-CoV-2) infection, with 3.9 million deaths $\left(2.17 \%\right.$ mortality rate). ${ }^{1}$ COVID-19 caused disruptions in many aspects of the health system, ${ }^{2}$ and systematic reviews suggested the high prevalence of COVID-19 is potentially associated with long-term burden. ${ }^{3,4}$ In addition, coinfection of SARS-CoV-2 and endemic infections in Asia is a major concern. ${ }^{5,6}$ As of June 30, 2021, 259,301 people in Thailand had been infected with COVID-19, and there were 2,023 deaths. $^{7}$ The most vulnerable group in the infected population was older people. SARS-CoV-2 infection in older people, particularly males, has been associated with more severe symptoms. ${ }^{8-11}$ In the United States, people aged 65 years and over accounted for approximately 80\% of all COVID-19 deaths. Individuals over age 85 years had 630 times the mortality rate of those aged 18-29 years. ${ }^{12}$ The Ministry of Public Health in Thailand reported death from SARS-CoV-2 among people over age 70 years was up to $12.1 \%$. $^{13}$

Many studies showed that COVID-19 vaccination decreased infection, hospitalization, and mortality rates. An observational study in Israel using national 
surveillance data found that the Pfizer-BioNTech mRNA vaccine BNT162b2 was $96.5 \%, 98.0 \%$, and $98.1 \%$ effective in preventing SARS-CoV-2 infection, hospitalization, and death, respectively. ${ }^{14} \mathrm{An}$ interim analysis of the Oxford-AstraZeneca ChAdOx1 nCoV-19 vaccine showed $70.4 \%$ efficacy in preventing SARS-CoV-2 infection. ${ }^{15} \mathrm{~A}$ study in Chile using an inactivated SARS-CoV-2 vaccine (CoronaVac) showed its effectiveness in preventing infection, hospitalization, and death was $65.9 \%, 90.3 \%$, and $86.3 \%$, respectively. ${ }^{16}$ Therefore, to successfully manage SARS-CoV-2 infection, countrywide COVID-19 vaccination is needed to achieve herd immunity.

The ChAdOx1 nCoV-19 and CoronaVac vaccinations have been the most commonly available vaccines in Thailand. The ChAdOx1 nCoV-19 vaccination is mostly given to people over age 60 years. However, some older people refuse to be vaccinated against COVID-19. The Strategic Advisory Group of Experts on Immunization from the World Health Organization (WHO) defined vaccine hesitancy as the delay in acceptance or refusal of vaccination despite availability of vaccination services. ${ }^{17,18}$ Factors that can influence vaccine hesitancy can be grouped into three categories: contextual, individual and group, and vaccine/vaccination-specific influences. ${ }^{17,18}$

A systematic review and meta-analysis of intended uptake and refusal of COVID-19 vaccines from 13 countries showed $60 \%$ of participants intended to get vaccinated, and $20 \%$ intended to refuse. ${ }^{19}$ Factors associated with vaccine refusal were being female, younger, having a lower income or education level, and being in an ethnic minority group. ${ }^{19}$ Despite older people benefiting the most from COVID-19 vaccination, few studies have investigated COVID-19 vaccine hesitancy in this group. To date, there has been no study on COVID-19 vaccine hesitancy in Thailand. Different social contexts, cultures, and politics may affect a person's intention to get vaccinated. Our study aimed to evaluate factors associated with COVID-19 vaccine hesitancy in Thai seniors. The findings will offer important insights for promoting vaccination among older people in Thailand.

\section{Materials and Methods}

\section{Setting and Study Design}

This study protocol was approved by the Human Research Ethics Committee of the Faculty of Medicine, Ramathibodi Hospital, Mahidol University (COA. MURA2021/441). We conducted a cross-sectional telephone survey about vaccine hesitancy in an outpatient geriatric clinic at Ramathibodi hospital. Over the last 2 years, this hospital had approximately 2,600 senior patients and 16,000 clinic visits. Patients aged 60 years and over who visited the geriatric clinic in the past 2 years were retrieved from the hospital database. These patients were contacted and invited to participate in this survey. Because we performed a telephone-based survey and because it was inconvenient for participants to sign written informed consent forms and manage documents during the pandemic, we did not obtain written informed consent. However, all participants gave verbal informed consent, which was recorded in accordance with the verbal informed consent protocol approved by the the Human Research Ethics Committee of the Faculty of Medicine, Ramathibodi Hospital, Mahidol University. Patients who agreed to participate were interviewed via telephone by a trained interviewer between June 20 and July 25, 2021. The survey was conducted in the Thai language. This study was conducted according to the Declaration of Helsinki and Good Clinical Practice guidelines. ${ }^{20,21}$

\section{Sample Size and Sampling}

The sample size was calculated with based on a $95 \%$ confidence level, 0.05 margin of error, assuming an observed proportion of respondents selecting a specific response option of $50 \%$, and finite correction for the study population of 2,600 patients. Using this method, the calculated sample size was 335 patients. The list of patients from the database was reordered using computerized randomization, and patients were contacted according to their order in the new randomized list.

\section{Questionnaire}

The questionnaire used in this study was self-developed following a review of the literature ${ }^{22-34}$ and agreement among experts. The questionnaire was structured into four sections covering sociodemographic data, medical history, COVID-19 pandemic-related information, and COVID-19 vaccine-related information. A pilot sample $(\mathrm{N}=10)$ was used to improve the language and clarity of expression of the survey items. The data from the pilot sample were not used in subsequent analyses. The final version of the questionnaire required around 30-45 minutes to complete. The questionnaire was originally developed in the Thai language. 


\section{Sociodemographic Characteristics and Medical History}

Participants' sociodemographic characteristics were obtained, including age, gender, ethnicity, marital status, education, employment status, current residence, monthly income, and income loss due to COVID-19. In addition, participants were asked to report their medical history, including their body mass index (BMI), ambulation, hearing problems, vision problems, history of smoking and drinking alcohol, food and drug allergies, history of previous vaccinations (including influenza, pneumococcal, zoster and diphtheria-tetanus-pertussis vaccines), underlying diseases (including diabetes, chronic kidney disease, respiratory disease, and psychiatric illness), subjective cognitive complaints, hospitalization in the past year, and overall health perception.

\section{COVID-19 Pandemic-Related Information}

Participants were asked about their knowledge about COVID-19, primary source of COVID-19 information, confidence in COVID-19 information from government and public health agencies, confidence in the Thailand healthcare system's ability to treat patients with COVID19, government measurements for controlling COVID-19 infection, risk for COVID-19 infection, self-perception about having severe COVID-19 infection, and attitude toward social distancing. Moreover, participants were asked if there was a patient with COVID in their neighborhood, if they knew anyone who had been infected with COVID-19, and if they knew anyone who had died from COVID-19.

\section{COVID-19 Vaccine-Related Information}

Participants were asked if they were hesitant to get a COVID-19 vaccine, which was defined as delay in acceptance or refusal of vaccination despite availability of vaccination services. They were asked if they knew anyone who had had a serious reaction to the COVID-19 vaccine, wanted to be vaccinated for COVID-19, thought the manufacturer of the COVID-19 vaccine influenced their decision to get vaccinated, and who they thought was the most desirable COVID-19 vaccine manufacturer. They were also asked whether they still wanted to get vaccinated if they were offered vaccines from a manufacturer they did not expect. Furthermore, those who were hesitant to receive a COVID-19 vaccine were asked why they were hesitant, and all participants were asked if they were willing to have the vaccine.

\section{Association Between Daily New COVID- 19 Cases and Vaccine Hesitancy}

Thailand was experiencing a new surge of COVID-19 infections at the time of our study, which was caused by a highly contagious Delta variant. ${ }^{35}$ We retrieved daily new COVID-19 patient data for Thailand from the WHO (COVID-19) official website ${ }^{36}$ to explore the association between the number of daily new cases and COVID-19 vaccine hesitancy.

\section{Statistical Analysis}

Nominal data (eg, presence of underlying diseases) were summarized as numbers and percentages. Continuous data (eg, age) were summarized as mean \pm standard deviation (SD), or median and interquartile range based on the normality of the distribution. Group comparisons were performed using chi-square or Fisher's tests for categorical variables and independent $t$-tests or Mann-Whitney $U$-tests for continuous parameters of continuous variables. Factors influencing vaccine hesitancy were investigated using binary logistic regression. Only variables that were statistically significant in the univariate logistic regression model were investigated in the multivariate logistic regression model. All statistical analyses were performed using SPSS version 26.0 for Windows (IBM Corp., Armonk, NY, USA). A p-value $<0.05$ was considered statistically significant.

\section{Results}

Of the 1,095 patients contacted, 282 (25.8\%) patients were able to participate in the interview and were enrolled in this study. The remaining $813(74.2 \%)$ patients were excluded from our study (Figure 1). In total, 125 (44.3\%) of the 282 participants had vaccine hesitancy, and 157 $(55.7 \%)$ indicated willingness to receive a vaccine.

\section{Participants' Sociodemographic Characteristics and Medical History}

Sociodemographic data are shown in Tables 1 and 2 . Participants were older adults aged 60-93 years (mean \pm SD age: $73.0 \pm 7.5$ years). Most participants were female (70.9\%), of Thai ethnicity (96.5\%), married (63.5\%), and lived in Bangkok (67.4\%). A comparison of characteristics between the vaccine hesitancy and vaccine acceptance 
2,589 patients were retrieved from hospital database

and re-sorted by computerized randomization

1,095 patients were called by the interviewers

282 eligible patients gave the interview by themselves

Figure I Study flow diagram.

groups showed that those who had an education level of elementary school or below had higher vaccine hesitancy than those who had graduated with a bachelor's degree or higher (odds ratio [OR] 2.56, 95\% confidence interval [CI]: 1.06-6.16, $\mathrm{p}=0.037$ ) (Table 3).

\section{COVID-19 Pandemic Related Information}

Table 4 presents the results for the items covering COVID19 pandemic-related information. Most participants $(62.0 \%)$ thought they knew quite a lot or a lot about COVID-19, and the majority (55.3\%) said their COVID19 information came from television or radio. Comparison of the vaccination hesitancy and vaccine acceptance groups showed that those who were not confident in the ability of the Thailand healthcare system to treat patients with COVID-19 were more likely to have vaccine hesitancy than those who were confident (OR 6.41, 95\% CI: 1.28-32.10, $\mathrm{p}=0.024)$ (Table 3).

\section{COVID-I 9 Vaccine-Related Information}

COVID-19 vaccine related information outcomes are depicted in Table 4. Most participants (89.3\%) did not know anyone who was severely allergic to the COVID19 vaccine, but $44.3 \%$ expressed vaccine hesitancy. Approximately one-third of participants (33.3\%) desired the Oxford-AstraZeneca ChAdOx1 nCoV-19 vaccine, followed by Moderna mRNA-1273 SARS-CoV-2 vaccine $(16.7 \%)$, and the Pfizer-BioNTech mRNA vaccine BNT162b2 (9.6\%) (Figure 2). The most common reasons for COVID-19 vaccine hesitancy were fear of COVID-19 vaccine-related adverse effects (35.2\%), possible complications caused by an underlying disease (26.4\%), and lack of confidence in COVID-19 vaccine efficacy or quality $(16.8 \%)$ (Figure 3$)$. The most important reasons for vaccination were that the COVID-19 vaccine could prevent severe COVID-19 infection or death (42.9\%) and protect them from COVID-19 infection (21.3\%), and that they were vulnerable group for COVID-19 infection (13.1\%) (Figure 4). The comparison of the vaccine hesitancy and vaccine acceptance groups showed that people who believed COVID-19 vaccine manufacturers influenced their decision to receive vaccination had higher vaccine hesitancy (OR 5.16, 95\% CI: 2.65-10.04, p<0.001). People who said they would reject vaccination if they were offered a vaccine from a manufacturer they had not heard of before had more vaccine hesitancy compared with those who accepted receiving different vaccines (OR 3.16, 95\% CI: 1.26-7.95, $\mathrm{p}=0.014$ ) (Table 3).

\section{Association Between Daily New COVID- 19 Cases and Vaccine Hesitancy}

On the first day of our survey (June 20, 2021), there were 3,682 new COVID-19 cases. The number of new cases per day had substantially increased by the end of this study (on July 22, 2021) to 15,355 patients per day. We categorized the number of daily new cases into four groups: $<5,000,5,000-7,499,7,500-9,999$, and $\geq 10,000$ cases per day (Table 4). We discovered that when there were $<5,000$ new cases per day, people were more 
Table I Baseline Characteristics of All Participants (N=282).

\begin{tabular}{|c|c|c|}
\hline Characteristics & $\mathbf{n}$ & $\%$ \\
\hline \multicolumn{3}{|l|}{ Age } \\
\hline 60-69 years & 99 & 35.1 \\
\hline 70-79 years & 123 & 43.6 \\
\hline $80+$ years & 60 & 21.3 \\
\hline \multicolumn{3}{|l|}{ Gender } \\
\hline Male & 82 & 29.1 \\
\hline Female & 200 & 70.9 \\
\hline \multicolumn{3}{|l|}{ Ethnicity } \\
\hline Thai & 273 & 96.5 \\
\hline Chinese & 9 & 3.2 \\
\hline \multicolumn{3}{|l|}{ Marital status } \\
\hline Single & 50 & 17.7 \\
\hline Married & 179 & 63.5 \\
\hline Divorced & 10 & 3.5 \\
\hline Widowed & 43 & 15.2 \\
\hline \multicolumn{3}{|l|}{ Education } \\
\hline Elementary school or below & 41 & 14.5 \\
\hline High school & 44 & 15.6 \\
\hline Bachelor's degree or higher & 197 & 69.9 \\
\hline \multicolumn{3}{|l|}{ Current residence } \\
\hline Bangkok & 190 & 67.4 \\
\hline Other provinces & 92 & 32.6 \\
\hline \multicolumn{3}{|l|}{ Employment } \\
\hline Retired & 233 & 82.6 \\
\hline Part-time job & 24 & 8.5 \\
\hline Full-time job & 25 & 8.9 \\
\hline \multicolumn{3}{|l|}{ Monthly income (baht) $(n=216)$} \\
\hline 10,000 or less & 53 & 18.8 \\
\hline $10,001-20,000$ & 52 & 18.4 \\
\hline $20,00 \mathrm{I}-30,000$ & 44 & 15.6 \\
\hline 30,001 or more & 67 & 23.8 \\
\hline \multicolumn{3}{|l|}{ Income loss due to COVID-19 } \\
\hline Yes & 53 & 18.8 \\
\hline No & 229 & 81.2 \\
\hline \multicolumn{3}{|l|}{ BMI $\left(\mathrm{kg} / \mathrm{m}^{2}\right)$} \\
\hline$<18.5$ & 12 & 4.3 \\
\hline $18.5-22.9$ & 111 & 39.4 \\
\hline $23-24.9$ & 71 & 25.2 \\
\hline $25-30$ & 68 & 24.1 \\
\hline$>30$ & 20 & 7.1 \\
\hline \multicolumn{3}{|l|}{ Ambulation } \\
\hline Gait aid & 14 & 5.0 \\
\hline Normal & 268 & 95.0 \\
\hline
\end{tabular}

(Continued)
Table I (Continued).

\begin{tabular}{|l|c|c|}
\hline Characteristics & $\mathbf{n}$ & $\%$ \\
\hline Hearing problem & 17 & \\
Hearing impairment & 265 & $9.0 \%$ \\
Normal & & \\
\hline Vision problem & 103 & 36.5 \\
Visual impairment & 179 & 63.5 \\
Normal & & \\
\hline History of smoking & 243 & 86.2 \\
No & 39 & 13.8 \\
Yes & & \\
\hline History of drinking alcohol & 256 & 90.8 \\
No & 26 & 9.2 \\
Yes & & \\
\hline Food allergy & 259 & 91.8 \\
No & 23 & 8.2 \\
Yes & & \\
\hline Drug allergy & 202 & 71.6 \\
No & 80 & 28.4 \\
Yes & & \\
\hline Vin & & \\
\hline
\end{tabular}

\begin{tabular}{|c|c|c|}
\hline Vaccination history & & \\
\hline Influenza vaccine & & \\
\hline No & 24 & 8.5 \\
\hline Yes & 258 & 91.5 \\
\hline Zoster vaccine & & \\
\hline No & 224 & 79.4 \\
\hline Yes & 58 & 20.6 \\
\hline Pneumococcal vaccine & & \\
\hline No & 172 & 61.0 \\
\hline Yes & 110 & 39.0 \\
\hline DTP vaccine & & \\
\hline No & 121 & 42.9 \\
\hline Yes & 161 & 57.1 \\
\hline Underlying disease & & \\
\hline Diabetes & 52 & 18.4 \\
\hline Chronic kidney disease & 11 & 3.9 \\
\hline Respiratory disease & 17 & 6.0 \\
\hline Psychiatric illness & 15 & 5.3 \\
\hline Subjective cognitive co & & \\
\hline No & 118 & 41.8 \\
\hline Yes & 164 & 58.2 \\
\hline Hospitalization in the & & \\
\hline No & 238 & 84.4 \\
\hline Yes & 44 & 15.6 \\
\hline
\end{tabular}

(Continued) 
Table I (Continued).

\begin{tabular}{|l|c|c|}
\hline Characteristics & $\mathbf{n}$ & $\%$ \\
\hline Overall health perception & & \\
Worst/bad & 7 & 2.5 \\
Average & 115 & 40.8 \\
Good/best & 160 & 56.7 \\
\hline
\end{tabular}

Abbreviations: BMI, body mass index; DTP, diphtheria-tetanus-pertussis. hesitant to get vaccinated than when there were $\geq 10,000$ new cases per day (OR 6.66, 95\% CI: 2.48-17.68, $\mathrm{p}<0.001$ ) (Table 3).

\section{Discussion}

The present study is one of the few available studies on COVID-19 vaccination hesitancy in older adults, and the

Table 2 Baseline Characteristics Associated with COVID-I9 Vaccine Hesitancy.

\begin{tabular}{|c|c|c|c|c|c|c|}
\hline \multirow[t]{2}{*}{ Characteristics } & \multicolumn{2}{|c|}{ Acceptance $(n=\mid 57)$} & \multicolumn{2}{|c|}{ Hesitancy $(n=125)$} & \multirow[t]{2}{*}{$\chi^{2}$} & \multirow[t]{2}{*}{ p-value } \\
\hline & $\mathbf{n}$ & $\%$ & $\mathbf{n}$ & $\%$ & & \\
\hline \multicolumn{7}{|l|}{ Age } \\
\hline $60-69$ years & 59 & 37.6 & 40 & 32.0 & 1.78 & 0.411 \\
\hline 70-79 years & 63 & 40.1 & 60 & 48.0 & & \\
\hline $80+$ years & 35 & 22.3 & 25 & 20.0 & & \\
\hline \multicolumn{7}{|l|}{ Gender } \\
\hline Male & 50 & 31.8 & 32 & 25.6 & 1.32 & 0.251 \\
\hline Female & 107 & 68.2 & 93 & 74.4 & & \\
\hline \multicolumn{7}{|l|}{ Ethnicity } \\
\hline Thai & 152 & 96.8 & 121 & 96.8 & 0 & 1 \\
\hline Chinese & 5 & 3.2 & 4 & 3.2 & & \\
\hline \multicolumn{7}{|l|}{ Marital status } \\
\hline Single & 27 & 17.2 & 23 & 18.4 & 0.6 & 0.898 \\
\hline Married & 98 & 62.4 & 81 & 64.8 & & \\
\hline Divorced & 6 & 3.8 & 4 & 3.2 & & \\
\hline Widowed & 26 & 16.6 & 17 & 13.6 & & \\
\hline \multicolumn{7}{|l|}{ Education } \\
\hline Elementary school or below & 16 & 10.2 & 25 & 20.0 & 5.96 & 0.051 \\
\hline High school & 28 & 17.8 & 16 & 12.8 & & \\
\hline Bachelor's degree or higher & 113 & 72.0 & 84 & 67.2 & & \\
\hline \multicolumn{7}{|l|}{ Current residence } \\
\hline Bangkok & 105 & 66.9 & 85 & 68.0 & 0.04 & 0.842 \\
\hline Other provinces & 52 & 33.1 & 40 & 32.0 & & \\
\hline \multicolumn{7}{|l|}{ Employment } \\
\hline Retired & 128 & 81.5 & 105 & 84.0 & 7.78 & 0.678 \\
\hline Part-time job & 13 & 8.3 & 11 & 8.8 & & \\
\hline Full-time job & 16 & 10.2 & 9 & 7.2 & & \\
\hline \multicolumn{7}{|l|}{ Income loss due to COVID } \\
\hline Yes & 29 & 18.5 & 24 & 19.2 & 0.02 & 0.876 \\
\hline No & 128 & 81.5 & 101 & 80.8 & & \\
\hline \multicolumn{7}{|c|}{ Monthly income (baht) $(n=2 \mid 6)$} \\
\hline 10,000 or less & 29 & 24.2 & 24 & 25.0 & 2.43 & 0.489 \\
\hline$|0,00|-20,000$ & 33 & 27.5 & 19 & 19.8 & & \\
\hline $20,001-30,000$ & 21 & 17.5 & 23 & 24.0 & & \\
\hline 30,001 or more & 37 & 30.8 & 30 & 31.3 & & \\
\hline
\end{tabular}

(Continued) 
Table 2 (Continued).

\begin{tabular}{|c|c|c|c|c|c|c|}
\hline \multirow[t]{2}{*}{ Characteristics } & \multicolumn{2}{|c|}{ Acceptance $(n=157)$} & \multicolumn{2}{|c|}{ Hesitancy $(n=125)$} & \multirow[t]{2}{*}{$\chi^{2}$} & \multirow[t]{2}{*}{ p-value } \\
\hline & $\mathbf{n}$ & $\%$ & $\mathbf{n}$ & $\%$ & & \\
\hline \multicolumn{7}{|l|}{ BMI $\left(\mathrm{kg} / \mathrm{m}^{2}\right)$} \\
\hline$<18.5$ & 8 & 5.1 & 4 & 3.2 & 1.68 & 0.794 \\
\hline $18.5-22.9$ & 62 & 39.5 & 49 & 39.2 & & \\
\hline $23-24.9$ & 41 & 26.1 & 30 & 24.0 & & \\
\hline $25-30$ & 37 & 23.6 & 31 & 24.8 & & \\
\hline$>30$ & 9 & 5.7 & 11 & 8.8 & & \\
\hline \multicolumn{7}{|l|}{ Ambulation } \\
\hline Gait aid & 5 & 3.2 & 9 & 7.2 & 2.38 & 0.123 \\
\hline Normal & 152 & 96.8 & 116 & 92.8 & & \\
\hline \multicolumn{7}{|l|}{ Hearing problem } \\
\hline Hearing impairment & 9 & 5.7 & 8 & 6.4 & 0.06 & 0.815 \\
\hline Normal & 148 & 94.3 & 117 & 93.6 & & \\
\hline \multicolumn{7}{|l|}{ Vision problem } \\
\hline Visual impairment & 57 & 36.3 & 46 & 36.8 & 0.01 & 0.932 \\
\hline Normal & 100 & 63.7 & 79 & 63.2 & & \\
\hline \multicolumn{7}{|l|}{ History of smoking } \\
\hline No & $|3|$ & 83.4 & 112 & 89.6 & 2.22 & 0.137 \\
\hline Yes & 26 & 16.6 & 13 & 10.4 & & \\
\hline \multicolumn{7}{|c|}{ History of drinking alcohol } \\
\hline No & 138 & 87.9 & 118 & 94.4 & 3.52 & 0.061 \\
\hline Yes & 19 & 12.1 & 7 & 5.6 & & \\
\hline \multicolumn{7}{|l|}{ Food allergy } \\
\hline No & 150 & 95.5 & 109 & 87.2 & 6.46 & 0.011 \\
\hline Yes & 7 & 4.5 & 16 & 12.8 & & \\
\hline \multicolumn{7}{|l|}{ Drug allergy } \\
\hline No & 116 & 73.9 & 86 & 68.8 & 0.89 & 0.347 \\
\hline Yes & $4 I$ & 26.1 & 39 & 31.2 & & \\
\hline \multicolumn{7}{|l|}{ Vaccination history } \\
\hline \multicolumn{7}{|l|}{ Influenza vaccine } \\
\hline No & 15 & 9.6 & 9 & 7.2 & 0.5 & 0.482 \\
\hline Yes & 142 & 90.4 & 116 & 92.8 & & \\
\hline \multicolumn{7}{|l|}{ Zoster vaccine } \\
\hline No & 127 & 80.9 & 97 & 77.6 & 0.46 & 0.497 \\
\hline Yes & 30 & 19.1 & 28 & 22.4 & & \\
\hline \multicolumn{7}{|c|}{ Pneumococcal vaccine } \\
\hline No & 96 & 61.1 & 76 & 60.8 & 0.004 & 0.953 \\
\hline Yes & 61 & 38.9 & 49 & 39.2 & & \\
\hline \multicolumn{7}{|l|}{ DTP vaccine } \\
\hline No & 62 & 39.5 & 59 & 47.2 & 1.69 & 0.194 \\
\hline Yes & 95 & 60.5 & 66 & 52.8 & & \\
\hline
\end{tabular}

(Continued) 
Table 2 (Continued).

\begin{tabular}{|c|c|c|c|c|c|c|}
\hline \multirow[t]{2}{*}{ Characteristics } & \multicolumn{2}{|c|}{ Acceptance $(n=157)$} & \multicolumn{2}{|c|}{ Hesitancy $(n=125)$} & \multirow[t]{2}{*}{$\chi^{2}$} & \multirow[t]{2}{*}{ p-value } \\
\hline & $\mathbf{n}$ & $\%$ & $\mathbf{n}$ & $\%$ & & \\
\hline \multicolumn{7}{|l|}{ Underlying disease } \\
\hline Diabetes & 25 & 15.9 & 27 & 21.6 & 1.49 & 0.222 \\
\hline Chronic kidney disease & 3 & 1.9 & 8 & 6.4 & 2.74 & 0.066 \\
\hline Respiratory disease & 7 & 4.5 & 10 & 8.0 & 1.54 & 0.215 \\
\hline Psychiatric illness & 6 & 3.8 & 9 & 7.2 & 1.58 & 0.209 \\
\hline \multicolumn{7}{|c|}{ Subjective cognitive complaint } \\
\hline No & 64 & 40.8 & 54 & 43.2 & 0.17 & 0.68 \\
\hline Yes & 93 & 59.2 & 71 & 56.8 & & \\
\hline \multicolumn{7}{|c|}{ Hospitalization in the past year } \\
\hline No & 136 & 86.6 & 102 & 81.6 & 1.33 & 0.248 \\
\hline Yes & 21 & 13.4 & 23 & 18.4 & & \\
\hline \multicolumn{7}{|c|}{ Overall health perception } \\
\hline Worst/bad & 2 & 1.3 & 5 & 4.0 & 3.02 & 0.221 \\
\hline Average & 61 & 38.9 & 54 & 43.2 & & \\
\hline Good/best & 94 & 59.9 & 66 & 52.8 & & \\
\hline
\end{tabular}

Abbreviations: BMI, body mass index; DTP, diphtheria-tetanus-pertussis.

first study on COVID-19 vaccine hesitancy in Thailand. We conducted a telephone survey of patients from the geriatric clinic at Ramathibodi Hospital, using information acquired from the hospital's database. Our survey was completed by 282 patients, of which $44.3 \%$ were hesitant to get vaccinated. Our study found that factors associated with high COVID-19 vaccine hesitancy were low education, lack of confidence in the healthcare system's ability to treat patients with COVID-19, vaccine manufacturers, being offered vaccines from an unexpected manufacturer, and a lower number of new COVID-19 cases per day. However, factors that influenced vaccine hesitancy in earlier studies $^{19,22,23,28,33,37-42}$ such as gender, income, marital status, and influenza vaccine history in the previous year, did not demonstrate associations in our study.

We found that people with an education level of elementary school or below had more vaccine hesitancy than those who had graduated with a bachelor's degree or higher. This finding was consistent with previous studies $^{22,25,40,41}$ from the United States, the United Kingdom, and Portugal. When compared with individuals who had confidence in the healthcare system's competence to manage patients with COVID-19, those who lacked confidence had 6.4 times higher vaccine hesitancy. A study from Portugal ${ }^{25}$ reported comparable results, and a Saudi Arabian study found that people who trusted their healthcare system were three times more likely to receive vaccines. $^{38}$

Vaccine manufacturers are one of Thailand's most controversial issues. Currently, only the ChAdOx1 nCoV-19 and CoronaVac vaccinations are available. Although the Delta variant was the COVID-19 variant most commonly of concern in Thailand, ${ }^{35}$ research is only available on the efficacy of ChAdOx1 nCoV-19 against Delta (effectiveness: $67 \%$, 95\% CI: $6.3-71.8) .{ }^{43}$ This could explain why the COVID-19 vaccine manufacturer influenced people's vaccine hesitancy. This was further supported by the finding from our study that people wanted to get vaccinated for two primary reasons: to prevent serious infection or death $(42.9 \%)$ and to prevent infection $(21.3 \%)$. To promote vaccination acceptability, the government and public health authorities should make high-potency COVID vaccine accessible to everyone. In addition, those who expected a certain vaccine but were instead given a different vaccine showed a higher rate of vaccine hesitancy. This could be a unique problem in the Thai situation, caused by the uncertain policies of the responsible agency. People's vaccine hesitancy could be reduced by open communication and a clear vaccine delivery strategy.

We observed that when the number of new COVID-19 infections grew, people's vaccine hesitancy decreased. Fear of COVID-19 was linked to risk perception in a previous investigation. ${ }^{44}$ Research suggested that those 
Table 3 Logistic Regression Results for COVID-19 Vaccine Hesitancy.

\begin{tabular}{|c|c|c|c|c|c|c|}
\hline \multirow[t]{2}{*}{ Variable } & \multicolumn{3}{|c|}{ Univariate } & \multicolumn{3}{|c|}{ Multivariate } \\
\hline & OR & $95 \% \mathrm{Cl}$ & p-value & aOR & $95 \% \mathrm{Cl}$ & p-value \\
\hline \multicolumn{7}{|l|}{ Education } \\
\hline Elementary school or below & 0.37 & $0.15-0.88$ & 0.025 & 2.56 & $1.06-6.16$ & 0.037 \\
\hline High school & 0.48 & $0.24-0.95$ & 0.034 & 0.7 & $0.30-1.65$ & 0.412 \\
\hline Bachelor's degree or higher & Ref & & & & & \\
\hline \multicolumn{7}{|l|}{ Food allergy } \\
\hline No & Ref & & & & & \\
\hline Yes & 0.32 & $0.13-0.80$ & 0.015 & 2.66 & $0.77-9.21$ & 0.122 \\
\hline \multicolumn{7}{|c|}{$\begin{array}{l}\text { How confident are you in government and public health agency } \\
\text { information on COVID-19? }\end{array}$} \\
\hline Not confident & 2.55 & $0.92-7.04$ & 0.072 & 1.06 & $0.28-4.07$ & 0.930 \\
\hline Quite not confident & 4.32 & $1.81-10.27$ & 0.001 & 3.05 & $0.96-9.72$ & 0.059 \\
\hline Quite confident & 1.69 & $0.78-3.64$ & 0.182 & 2.07 & $0.74-5.81$ & 0.169 \\
\hline Confident & Ref & & & & & \\
\hline \multicolumn{7}{|c|}{$\begin{array}{l}\text { How confident are you in Thailand's healthcare system's ability to } \\
\text { treat patients with COVID-19? }\end{array}$} \\
\hline Not confident & 6.22 & $1.62-23.93$ & 0.008 & 6.41 & $1.28-32.10$ & 0.024 \\
\hline Quite not confident & 1.81 & $0.79-4.13$ & 0.159 & 1.14 & $0.38-3.44$ & 0.816 \\
\hline Quite confident & 1.33 & $0.78-2.28$ & 0.301 & 0.79 & $0.38-1.63$ & 0.517 \\
\hline Confident & Ref & & & & & \\
\hline \multicolumn{7}{|c|}{ What is your risk of getting COVID-I9? } \\
\hline Very low risk & Ref & & & & & \\
\hline Low risk & 1.55 & $0.75-3.23$ & 0.237 & 1.56 & $0.56-4.29$ & 0.393 \\
\hline High risk & 2.88 & $1.29-6.44$ & 0.01 & 2.57 & $0.83-7.99$ & 0.103 \\
\hline Very high risk & 2.79 & $0.90-8.69$ & 0.077 & 3.05 & $0.68-13.75$ & 0.147 \\
\hline \multicolumn{7}{|c|}{$\begin{array}{l}\text { What is your chance of having severe COVID-19 infection or life- } \\
\text { threatening condition if you get COVID-19 infection? }\end{array}$} \\
\hline Very low chance & Ref & & & & & \\
\hline Low chance & 2.44 & $0.99-6.06$ & 0.054 & 1.76 & $0.56-5.51$ & 0.332 \\
\hline High chance & 3.06 & $1.18-7.90$ & 0.021 & 1.57 & $0.46-5.42$ & 0.472 \\
\hline Very high chance & 6.21 & $1.93-19.99$ & 0.002 & 1.76 & $0.38-8.12$ & 0.467 \\
\hline \multicolumn{7}{|c|}{$\begin{array}{l}\text { Do you think the manufacturer of the COVID-19 vaccine influenced } \\
\text { your decision to get vaccinated? }\end{array}$} \\
\hline No & Ref & & & & & \\
\hline Yes & 6.20 & $3.69-10.43$ & $<0.001$ & 5.16 & $2.65-10.04$ & $<0.001$ \\
\hline \multicolumn{7}{|c|}{$\begin{array}{l}\text { Do you want to get vaccinated if you are offered a vaccine from a } \\
\text { manufacturer that you did not expect? }\end{array}$} \\
\hline No & 5.20 & $2.50-10.85$ & $<0.001$ & 3.16 & $1.26-7.95$ & 0.014 \\
\hline Yes & Ref & & & & & \\
\hline Unsure & 4.29 & $2.20-8.38$ & $<0.001$ & 1.95 & $0.82-4.67$ & 0.133 \\
\hline \multicolumn{7}{|c|}{ Number of new COVID-19 patients per day (patients) } \\
\hline Less than 5000 & 3.89 & $1.77-8.54$ & 0.001 & 6.62 & $2.48-17.68$ & $<0.001$ \\
\hline $5000-7499$ & 1.26 & $0.68-2.33$ & 0.465 & 1.61 & $0.74-3.54$ & 0.233 \\
\hline $7500-9999$ & 0.79 & $0.40-1.55$ & 0.493 & 0.77 & $0.33-1.81$ & 0.554 \\
\hline More than 10,000 & Ref & & & & & \\
\hline
\end{tabular}

Abbreviations: OR, odds ratio; aOR, adjusted odds ratio; $\mathrm{Cl}$, confidence interval; Ref, reference group. 
Table 4 COVID-19 Pandemic- and Vaccine-Related Factors Associated with COVID-19 Vaccine Hesitancy.

\begin{tabular}{|c|c|c|c|c|c|c|}
\hline \multirow[t]{2}{*}{ COVID-19 Pandemic and Vaccine Related Information } & \multicolumn{2}{|c|}{ Acceptance $(n=157)$} & \multicolumn{2}{|c|}{ Hesitancy $(n=125)$} & \multirow[t]{2}{*}{$\chi^{2}$} & \multirow[t]{2}{*}{ p-value } \\
\hline & $\mathbf{n}$ & $\%$ & $\mathbf{n}$ & $\%$ & & \\
\hline \multicolumn{7}{|l|}{ How much do you know about COVID-I9? } \\
\hline Nothing & 5 & 3.2 & 7 & 5.6 & 1.45 & 0.694 \\
\hline Little & 51 & 32.5 & 44 & 35.2 & & \\
\hline Quite a lot & 89 & 56.7 & 66 & 52.8 & & \\
\hline A lot & 12 & 7.6 & 8 & 6.4 & & \\
\hline \multicolumn{7}{|l|}{ What is your primary source of COVID-19 information? } \\
\hline Television, radio & 85 & 54.1 & 71 & 56.8 & 4.16 & 0.527 \\
\hline Newspaper & 2 & 1.3 & I & 0.8 & & \\
\hline Friend & 12 & 7.6 & 9 & 7.2 & & \\
\hline News website & 15 & 9.6 & 9 & 7.2 & & \\
\hline Social network & 39 & 24.8 & 35 & 28.2 & & \\
\hline Others & 4 & 2.5 & 0 & 0.0 & & \\
\hline \multicolumn{7}{|c|}{$\begin{array}{l}\text { How confident are you in government and public health agency } \\
\text { information on COVID-19? }\end{array}$} \\
\hline Not confident & 14 & 8.9 & 14 & 11.2 & 14.37 & 0.002 \\
\hline Quite not confident & 23 & 14.6 & 39 & 31.2 & & \\
\hline Quite confident & 92 & 58.6 & 61 & 48.8 & & \\
\hline Confident & 28 & 17.8 & 11 & 8.8 & & \\
\hline \multicolumn{7}{|c|}{$\begin{array}{l}\text { How confident are you in Thailand's healthcare system's ability to } \\
\text { treat patients with COVID-19? }\end{array}$} \\
\hline No confident & 3 & 1.9 & 11 & 8.8 & 9.22 & 0.026 \\
\hline Quite not confident & 15 & 9.6 & 16 & 12.8 & & \\
\hline Quite confident & 83 & 52.9 & 65 & 52.0 & & \\
\hline Confident & 56 & 35.7 & 33 & 26.4 & & \\
\hline \multicolumn{7}{|c|}{$\begin{array}{l}\text { How effective are the government's measurements for controlling } \\
\text { COIVD-19 infection? }\end{array}$} \\
\hline Insufficient & 50 & 31.8 & 46 & 36.8 & 3.01 & 0.39 \\
\hline Quite insufficient & 45 & 28.7 & 42 & 33.6 & & \\
\hline Quite sufficient & 51 & 32.5 & 30 & 24.0 & & \\
\hline Sufficient & 11 & 7.0 & 7 & 5.6 & & \\
\hline \multicolumn{7}{|l|}{ What is your risk of getting COVID-19 infection? } \\
\hline Very low risk & 29 & 18.5 & 13 & 10.4 & 8.77 & 0.033 \\
\hline Low risk & 89 & 56.7 & 62 & 49.6 & & \\
\hline High risk & 31 & 19.7 & 40 & 32.0 & & \\
\hline Very high risk & 8 & 5.1 & 10 & 8.0 & & \\
\hline \multicolumn{7}{|c|}{$\begin{array}{l}\text { What is your chance of having severe COVID-19 infection or life- } \\
\text { threatening condition if you get COVID-19 infection? }\end{array}$} \\
\hline Very low chance & 23 & 14.6 & 7 & 5.6 & 10.69 & 0.014 \\
\hline Low chance & 82 & 52.2 & 61 & 48.8 & & \\
\hline High chance & 43 & 27.4 & 40 & 32.0 & & \\
\hline Very high chance & 9 & 5.7 & 17 & 13.6 & & \\
\hline \multicolumn{7}{|c|}{$\begin{array}{l}\text { How uneasy/anxious/agitated/sad/worried do you feel when you } \\
\text { have to practice social distancing? }\end{array}$} \\
\hline Never & 112 & 71.3 & 76 & 60.8 & 3.91 & 0.271 \\
\hline Sometimes & 36 & 22.9 & 39 & 31.2 & & \\
\hline Often & 5 & 3.2 & 7 & 5.6 & & \\
\hline
\end{tabular}

(Continued) 
Table 4 (Continued).

\begin{tabular}{|c|c|c|c|c|c|c|}
\hline \multirow[t]{2}{*}{ COVID-19 Pandemic and Vaccine Related Information } & \multicolumn{2}{|c|}{ Acceptance $(n=157)$} & \multicolumn{2}{|c|}{ Hesitancy $(n=125)$} & \multirow[t]{2}{*}{$\chi^{2}$} & \multirow[t]{2}{*}{ p-value } \\
\hline & $\mathbf{n}$ & $\%$ & $\mathbf{n}$ & $\%$ & & \\
\hline Always & 4 & 2.5 & 3 & 2.4 & & \\
\hline \multicolumn{7}{|l|}{ Is there a patient with COVID-19 in your neighborhood? } \\
\hline No & 86 & 54.8 & 58 & 46.4 & 1.95 & 0.162 \\
\hline Yes & 71 & 45.2 & 67 & 53.6 & & \\
\hline \multicolumn{7}{|l|}{ Do you know anyone who has been infected with COVID-I9? } \\
\hline No & 97 & 61.8 & 63 & 50.4 & 3.67 & 0.055 \\
\hline Yes & 60 & 38.2 & 62 & 49.6 & & \\
\hline \multicolumn{7}{|l|}{ Do you know anyone who has died as a result of COVID-19? } \\
\hline No & 130 & 82.8 & 97 & 77.6 & 1.2 & 0.273 \\
\hline Yes & 27 & 17.2 & 28 & 22.4 & & \\
\hline \multicolumn{7}{|l|}{$\begin{array}{l}\text { Do you know anyone who has had a serious reaction to the } \\
\text { COVID-19 vaccine? }\end{array}$} \\
\hline No & 145 & 92.4 & 107 & 85.6 & 3.34 & 0.068 \\
\hline Yes & 12 & 7.6 & 18 & 14.4 & & \\
\hline \multicolumn{7}{|l|}{ Will you refuse COVID-19 vaccination? } \\
\hline No & $15 \mid$ & 96.2 & 118 & 94.4 & 0.5 & 0.479 \\
\hline Yes & 6 & 3.8 & 7 & 5.6 & & \\
\hline \multicolumn{7}{|l|}{$\begin{array}{l}\text { Do you think the manufacturers of the COVID-19 vaccine } \\
\text { influenced your decision to get vaccinated? }\end{array}$} \\
\hline No & 118 & 75.2 & 41 & 32.8 & 50.78 & $<0.001$ \\
\hline Yes & 39 & 24.8 & 84 & 67.2 & & \\
\hline \multicolumn{7}{|c|}{$\begin{array}{l}\text { Do you want to get vaccinated if you are offered a vaccine from a } \\
\text { manufacturer you did not expect? }\end{array}$} \\
\hline No & 12 & 7.6 & 30 & 24.0 & 33.9 & $<0.001$ \\
\hline Yes & 129 & 82.2 & 62 & 49.6 & & \\
\hline Unsure & 16 & 10.2 & 33 & 26.4 & & \\
\hline \multicolumn{7}{|l|}{ Number of new COVID-19 patients per day (patients) } \\
\hline Less than 5000 & 13 & 8.3 & 32 & 25.6 & 17.47 & 0.001 \\
\hline $5000-7499$ & 49 & 31.2 & 39 & 31.2 & & \\
\hline $7500-9999$ & 46 & 29.3 & 23 & 18.4 & & \\
\hline More than 10,000 & 49 & 31.2 & 31 & 24.8 & & \\
\hline
\end{tabular}

who perceived a greater risk of infection were more likely to receive vaccines. ${ }^{25,33}$ We hypothesized that as the number of new COVID-19 infections rose, so did the risk of becoming infected, which resulted in less vaccination hesitancy.

Some previous studies that suggested females had higher COVID-19 vaccine hesitancy; ${ }^{22,37,39,42}$ however, we were unable to replicate this finding. In addition, we found no link between income and COVID-19 vaccine hesitancy, although several previous studies demonstrated people with higher income had lower vaccine hesitancy. $^{28,40,42}$ Our study found no relationship between marital status and COVID-19 vaccine hesitancy. However, in some previous research, married people exhibited less COVID-19 vaccine hesitancy. ${ }^{33,38}$ This may be related to the fact that our participants were older individuals with different marital status than younger groups. Furthermore, unlike some previous research, ${ }^{25,28,33,41}$ we were unable to show that those who had had an influenza vaccine injection in the previous year had less COVID-19 vaccine hesitancy. This could be because all older patients in our geriatric clinic 
Oxford/AstraZeneca vaccine

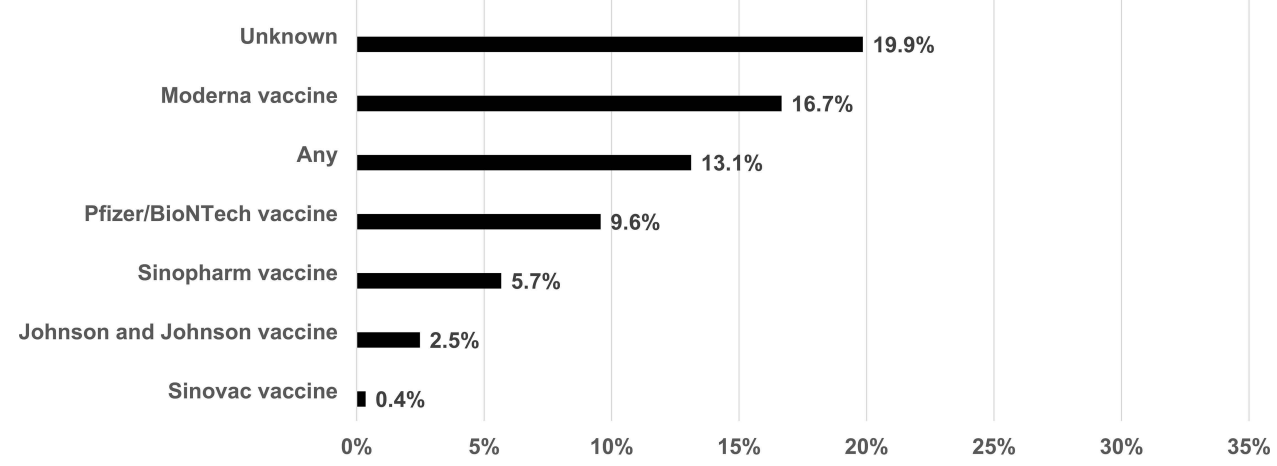

Oxford/AstraZeneca: Oxford-AstraZeneca ChAdOx1 nCoV-19 vaccine Moderna Moderna: mRNA-1273 SARS-CoV-2 vaccine

Pfizer-BioNTech: Pfizer-BioNTech mRNA vaccine BNT162b2

Sinopharm: Inactivated SARS-CoV-2 vaccine (BBIBP-CorV)

Johnson and Johnson: Janssen Ad26.COV2.S vaccine

Sinovac: Inactivated SARS-CoV-2 vaccine (CoronaVac)

Figure 2 Most desirable vaccine manufacturer as perceived by participants.

I'm concerned about the vaccine's potential adverse effects. I have underlying medical conditions that could make the vaccination unsafe.

I'm not confident about vaccine efficacy and quality.

The news regarding COVID-19 has been exaggeratedly terrible.

I'm delaying vaccination because l'd like to observe potential adverse effects from others.

I would like to wait for a vaccine that is currently unavailable.

I believe my chances of developing a severe infection are low.

I believe I was unable to detect my abnormal symptom as a result of vaccination.

I have a low risk of getting infection because I rarely go outside or interact with others.

I believe I could sufficiently protect myself from infection. $0.8 \%$

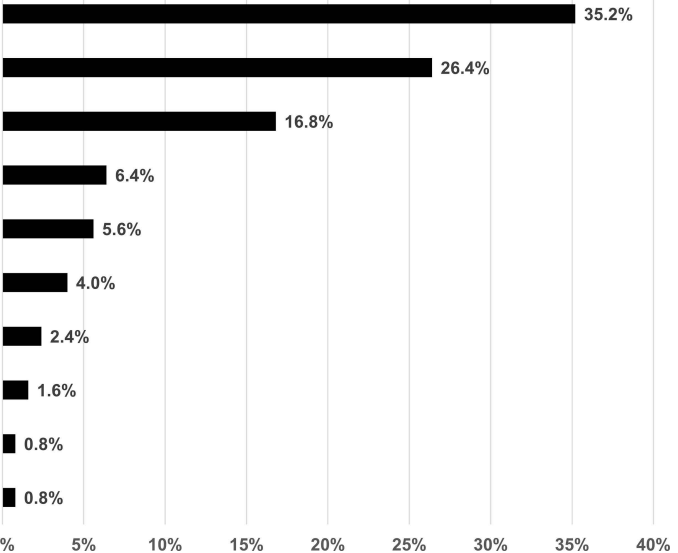

Figure 3 Most important reason for COVID-19 vaccine hesitancy.

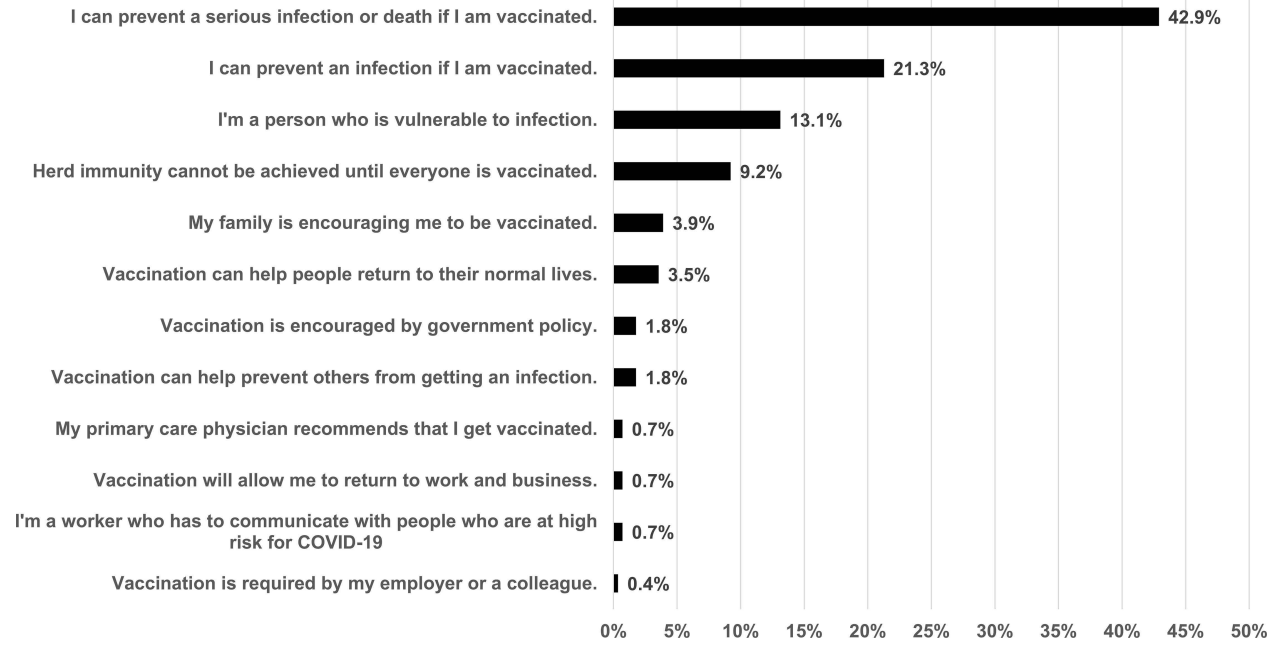

Figure 4 Most important reasons to get vaccinated against COVID-I9. 
were encouraged to get an annual influenza vaccine, with vaccination rates reaching $91.5 \%$.

Our study was one of only a few studies on vaccine hesitancy that employed direct data gathering rather than an online volunteer survey. As a result, we were able to enroll diverse people in our study, including older adults who were unable to use technological devices. We began our research at a time when the number of COVID-19 patients was growing exponentially because of the Delta variant, so we could compare the incidence of COVID-19 vaccination hesitancy based on daily new cases. Furthermore, the majority of COVID-19 vaccine hesitancy studies were conducted while vaccinations were not generally available, whereas our study examined people's attitudes after vaccines became widely accessible.

Our study had several limitations. We recruited participants from a geriatric clinic, most of whom lived in Bangkok, and the results should be interpreted with caution in other settings. Only one-fourth of those who were contacted were able to participate in this study and complete the questionnaires on their own; therefore the number of participants was fewer than planned. We were unaware that some participants would refuse to answer questions on sensitive subjects such as their incomes, and therefore were missing some information. Finally, although we demonstrated associations between higher COVID-19 vaccine hesitancy and low education level, lack of confidence in the healthcare system's ability to treat patients with COVID-19, vaccine manufacturers, being offered vaccines from an unexpected manufacturer, and a lower number of new COVID-19 cases per day, we could not demonstrate causality.

\section{Conclusion}

The prevalence of COVID-19 vaccine hesitancy among Thai seniors is relatively high. Low education level, lack of confidence in the healthcare system's ability to treat patients with COVID-19, vaccine manufacturers, being offered vaccines from an unexpected manufacturer, and a lower number of new daily COVID cases are linked to greater COVID-19 vaccine hesitancy. These findings will help in promoting COVID-19 vaccination among Thailand's senior citizens.

\section{Ethics Approval and Informed Consent}

The study protocol was approved by the Human Research Ethics Committee of the Faculty of Medicine, Ramathibodi Hospital, Mahidol University (COA.
MURA2021/441). This study was conducted according to the Declaration of Helsinki and Good Clinical Practice guidelines. Because we performed a telephone-based survey and it was inconvenient for participants to sign written informed consent and manage documents during the pandemic, we did not receive written informed consent. However, all participants provided verbal informed consent, which was recorded.

\section{Acknowledgments}

We would like to thank Piangporn Charernwat for assistance with data collection and project coordination, Krittika Saranburut for statistical analysis advice, and all interviewers and participants.

\section{Author Contributions}

All authors made a significant contribution to the work reported, whether that is in the conception, study design, execution, acquisition of data, analysis and interpretation, or in all these areas; took part in drafting, revising or critically reviewing the article; gave final approval of the version to be published; have agreed on the journal to which the article has been submitted; and agree to be accountable for all aspects of the work.

\section{Funding}

This research project was supported by the Faculty of Medicine, Ramathibodi Hospital, Mahidol University.

\section{Disclosure}

The authors declare that they have no conflicts of interest for this work.

\section{References}

1. World Health Organization. WHO Coronavirus (COVID-19) DASHBOARD. Available from: https://covid19.who.int/. Accessed July 24, 2021

2. Fahriani M, Anwar S, Yufika A, et al. Disruption of childhood vaccination during the COVID-19 pandemic in Indonesia. Narra J. 2021;1 (1):e7. doi:10.52225/narraj.v1i1.7

3. Yusuf F, Fahriani M, Mamada SS, et al. Global prevalence of prolonged gastrointestinal symptoms in COVID-19 survivors and potential pathogenesis: a systematic review and meta-analysis. F1000Res. 2021;10:301. doi:10.12688/f1000research.52216.1

4. Fahriani M, Ilmawan M, Fajar JK, et al. Persistence of long COVID symptoms in COVID-19 survivors worldwide and its potential pathogenesis - a systematic review and meta-analysis. Narra J. 2021;1(2): e36. doi:10.52225/narraj.v1i2.36

5. Masyeni S, Santoso MS, Widyaningsih PD, et al. Serological crossreaction and coinfection of dengue and COVID-19 in Asia: experience from Indonesia. Int $J$ Infect Dis. 2021;102:152-154. doi:10.1016/j. ijid.2020.10.043 
6. Bastola A, Sah R, Rajbhandari SK, et al. SARS-CoV-2 and orientia tsutsugamushi co-infection in a young teen, Nepal: significant burden in limited-resource countries in Asia? Narra J. 2021;1(2):e34. doi:10.52225/narraj.v1i2.34

7. World Health Organization. Thailand situation. Available from: https://covid19.who.int/region/searo/country/th. Accessed July 24, 2021.

8. Yanez ND, Weiss NS, Romand JA, Treggiari MM. COVID-19 mortality risk for older men and women. BMC Public Health. 2020;20 (1):1742. doi:10.1186/s12889-020-09826-8

9. Ward H, Atchison C, Whitaker M, et al. SARS-CoV-2 antibody prevalence in England following the first peak of the pandemic. Nat Commun. 2021;12(1):905. doi:10.1038/s41467-021-21237-w

10. Pastor-Barriuso R, Perez-Gomez B, Hernan MA, et al. Infection fatality risk for SARS-CoV-2 in community dwelling population of Spain: nationwide seroepidemiological study. BMJ. 2020;371:m4509. doi:10.1136/bmj.m4509

11. O'Driscoll M, Ribeiro Dos Santos G, Wang L, et al. Age-specific mortality and immunity patterns of SARS-CoV-2. Nature. 2021;590 (7844):140-145. doi:10.1038/s41586-020-2918-0

12. Centers for Disease Control and Prevention (CDC). COVID-19 guidance for older adults. Available from: https://www.cdc.gov/aging/ covid19-guidance.html. Accessed July 24, 2021.

13. World Health Organization. Coronavirus disease 2019 (COVID-19) WHO Thailand situation report - 17 April 2020. Available from: https://www.who.int/docs/default-source/searo/thailand/2020-04-17tha-sitrep-55-covid19-final.pdf?sfvrsn $=$ c59388b9_0. Accessed July 24, 2021.

14. Haas EJ, Angulo FJ, McLaughlin JM, et al. Impact and effectiveness of mRNA BNT162b2 vaccine against SARS-CoV-2 infections and COVID-19 cases, hospitalisations, and deaths following a nationwide vaccination campaign in Israel: an observational study using national surveillance data. Lancet. 2021;397(10287):1819-1829. doi:10.1016/ S0140-6736(21)00947-8

15. Voysey M, Clemens SAC, Madhi SA, et al. Safety and efficacy of the ChAdOx1 nCoV-19 vaccine (AZD1222) against SARS-CoV-2: an interim analysis of four randomised controlled trials in Brazil, South Africa, and the UK. Lancet. 2021;397(10269):99-111. doi:10.1016/S0140-6736(20)32661-1

16. Jara A, Undurraga EA, Gonzalez C, et al. Effectiveness of an inactivated SARS-CoV-2 vaccine in Chile. $N$ Engl J Med. 2021;385 (10):875-884. doi:10.1056/NEJMoa2107715

17. Strategic Advisory Group of Experts (SAGE) on Immunization. Report of the SAGE working group on vaccine hesitancy. Available from: https://www.who.int/immunization/sage/meetings/2014/octo ber/1_Report_WORKING_GROUP_vaccine_hesitancy_final.pdf. Accessed July 24, 2021.

18. MacDonald NE. Hesitancy SWGoV. Vaccine hesitancy: definition, scope and determinants. Vaccine. 2015;33(34):4161-4164. doi:10.10 16/j.vaccine.2015.04.036

19. Robinson E, Jones A, Lesser I, Daly M. International estimates of intended uptake and refusal of COVID-19 vaccines: a rapid systematic review and meta-analysis of large nationally representative samples. Vaccine. 2021;39(15):2024-2034. doi:10.1016/j.vaccine.2021.02.005

20. World Medical Association. World Medical Association declaration of Helsinki: ethical principles for medical research involving human subjects. JAMA. 2013;310(20):2191-2494. doi:10.1001/jama.2013.28 1053

21. World Medical Association. WMA declaration of Helsinki - ethical principles for medical research involving human subjects. Available from: https://www.wma.net/policies-post/wma-declaration-of-hel sinki-ethical-principles-for-medical-research-involving-human-sub jects/. Accessed August 1, 2021.

22. Robertson E, Reeve KS, Niedzwiedz CL, et al. Predictors of COVID19 vaccine hesitancy in the UK household longitudinal study. Brain Behav Immun. 2021;94:41-50. doi:10.1016/j.bbi.2021.03.008
23. Lin C, Tu P, Beitsch LM. Confidence and receptivity for COVID-19 vaccines: a rapid systematic review. Vaccines. 2020;9(1):16. doi:10. 3390/vaccines 9010016

24. Okubo R, Yoshioka T, Ohfuji S, Matsuo T, Tabuchi T. COVID-19 vaccine hesitancy and its associated factors in Japan. Vaccines. 2021;9(6):662. doi:10.3390/vaccines9060662

25. Soares P, Rocha JV, Moniz M, et al. Factors associated with COVID19 vaccine hesitancy. Vaccines. 2021;9(3):300. doi:10.3390/vaccines 9030300

26. Fridman A, Gershon R, Gneezy A, Capraro V. COVID-19 and vaccine hesitancy: a longitudinal study. PLoS One. 2021;16(4): e0250123. doi:10.1371/journal.pone.0250123

27. Kreps S, Prasad S, Brownstein JS, et al. Factors associated with US adults' likelihood of accepting COVID-19 vaccination. JAMA Netw Open. 2020;3 (10):e2025594. doi:10.1001/jamanetworkopen.2020.25594

28. Kelly BJ, Southwell BG, McCormack LA, et al. Predictors of willingness to get a COVID-19 vaccine in the U.S. BMC Infect Dis. 2021;21(1):338. doi:10.1186/s12879-021-06023-9

29. Syed Alwi SAR, Rafidah E, Zurraini A, Juslina O, Brohi IB, Lukas S. A survey on COVID-19 vaccine acceptance and concern among Malaysians. BMC Public Health. 2021;21(1):1129. doi:10.1186/ s12889-021-11071-6

30. Daly M, Robinson E. Willingness to vaccinate against COVID-19 in the U.S.: representative longitudinal evidence from April to October 2020. Am J Prev Med. 2021;60(6):766-773. doi:10.1016/j.amepre. 2021.01.008

31. Loomba S, de Figueiredo A, Piatek SJ, de Graaf K, Larson HJ. Measuring the impact of COVID-19 vaccine misinformation on vaccination intent in the UK and USA. Nat Hum Behav. 2021;5(3):337348. doi:10.1038/s41562-021-01056-1

32. Callaghan T, Moghtaderi A, Lueck JA, et al. Correlates and disparities of intention to vaccinate against COVID-19. Soc Sci Med. 2021;272:113638. doi:10.1016/j.socscimed.2020.113638

33. Wang J, Jing R, Lai X, et al. Acceptance of COVID-19 vaccination during the COVID-19 pandemic in China. Vaccines. 2020;8(3):482. doi:10.3390/vaccines 8030482

34. Ward JK, Alleaume C, Peretti-Watel P, Group C. The French public's attitudes to a future COVID-19 vaccine: the politicization of a public health issue. Soc Sci Med. 2020;265:113414. doi:10.1016/j. socscimed.2020.113414

35. World Health Organization. Coronavirus disease 2019 (COVID19) WHO Thailand situation report - 22 July 2021. Available from: https://cdn.who.int/media/docs/default-source/searo/thai land/2021_07_22_eng-sitrep-193-covid19.pdf?sfvrsn=a0fdd5a7 3. Accessed August 1, 2021.

36. World Health Organization. WHO Coronavirus (COVID-19) dashboard (Thailand). Available from: https:/covid19.who.int/region/ searo/country/th. Accessed August 1, 2021.

37. Neumann-Bohme S, Varghese NE, Sabat I, et al. Once we have it, will we use it? A European survey on willingness to be vaccinated against COVID-19. Eur J Health Econ. 2020;21(7):977-982. doi:10.1007/s10198-020-01208-6

38. Al-Mohaithef M, Padhi BK. Determinants of COVID-19 vaccine acceptance in Saudi Arabia: a web-based national survey. $J$ Multidiscip Healthc. 2020;13:1657-1663. doi:10.2147/JMDH. S276771

39. Yoda T, Katsuyama H. Willingness to receive COVID-19 vaccination in Japan. Vaccines. 2021;9(1):48. doi:10.3390/vaccines9010048

40. Williams L, Flowers P, McLeod J, Young D, Rollins L; The Catalyst Project T. Social patterning and stability of intention to accept a COVID-19 vaccine in Scotland: will those most at risk accept a vaccine? Vaccines. 2021;9(1):48. doi:10.3390/vaccines9010017

41. Fisher KA, Bloomstone SJ, Walder J, Crawford S, Fouayzi H, Mazor KM. Attitudes toward a potential SARS-CoV-2 vaccine: a survey of U.S. adults. Ann Intern Med. 2020;173(12):964-973. doi:10.7326/ M20-3569 
42. Reiter PL, Pennell ML, Katz ML. Acceptability of a COVID-19 vaccine among adults in the United States: how many people would get vaccinated? Vaccine. 2020;38(42):6500-6507. doi:10.1016/j. vaccine.2020.08.043

43. Lopez Bernal J, Andrews N, Gower C, et al. Effectiveness of COVID-19 vaccines against the B.1.617.2 (delta) variant. $N$ Engl $J$ Med. 2021;385(7):585-594. doi:10.1056/NEJMoa2108891
44. Han MFY, Mahendran R, Yu J. Associations between fear of COVID-19, affective symptoms and risk perception among community-dwelling older adults during a COVID-19 lockdown. Front Psychol. 2021;12:638831. doi:10.3389/fpsyg.2021.638831

\section{Publish your work in this journal}

Patient Preference and Adherence is an international, peer-reviewed, open access journal that focusing on the growing importance of patient preference and adherence throughout the therapeutic continuum. Patient satisfaction, acceptability, quality of life, compliance, persistence and their role in developing new therapeutic modalities and compounds to optimize clinical outcomes for existing disease states are major areas of interest for the journal. This journal has been accepted for indexing on PubMed Central. The manuscript management system is completely online and includes a very quick and fair peer-review system, which is all easy to use. Visit http:// www.dovepress.com/testimonials.php to read real quotes from published authors. 